\title{
Book review: Pat Bazeley’s ‘A practical introduction to Mixed Methods for Business and Management'
}

\author{
Review by EJBRM Editor, Ann Brown \\ DOI: 10.34190/JBRM.18.1.005
}

\author{
A Practical Introduction to Mixed Methods for Business \& Management \\ Author: Pat Bazeley \\ Publisher: Sage, Los Angeles \\ Year of publication: 2019
}

\begin{abstract}
Mixed Methods is a research methodology that integrates several types of data (both qualitative and quantitative) for a research project, using more than one analytical technique. This book gives an excellent introduction to the methodology, focusing on the issues involved in the handling of multiple approaches. The readership is assumed to have experience of business research and at least the skill to apply some of the current research methodologies. A mixed methods researcher needs not only an awareness of the main research methodologies, but also the ability to learn how to use them or has access to collaborators who do have the appropriate complementary expertise.
\end{abstract}

Mixed methods is a particularly powerful method for business and management research. Business problems typically involve specialist discipline facets embedded in and significantly affected by the social context. In such a situation both the hard quantitative data measuring performance and the qualitative information explaining the community responses can throw light on the problem and it's interpretation. In chapter 1 , the author makes a strong case for the great potential value of combining more than one research method. As she states (P13), using these methods 'to assess the same or different facets of an entity or an experience can lead to enriched, elaborated and/or expanded understanding of that phenomenon'. The effective integration of the insights developed from each and every approach used is needed to obtain this value.

Chapter 2 is the core of the book. It addresses the question as to whether mixed methods is appropriate for your research problem. The five component model (figure 2.1) offers an excellent overview of the major elements of any research project design. This model suggests that the final decision to adopt (or not to adopt) mixed methods comes when the three elements of goals (setting the direction of the research), conceptual framework (for the research subject) and research questions (actionable and specific) have been clarified.

A valuable feature of this book is the use of such wonderful case examples. As each key concept or activity is introduced a case example accompanies it as illustration. The author uses a research project carried out by herself to show how each of the steps described for developing a mixed methods study might work out in practice. Indeed the book greatly benefits from the long-time personal experience of the author in using this type of research approach.

The book has an easy to read writing style. The five chapters follow a well thought out logical sequence of topics. Chapter1 establishes what can be achieved with mixed methods. Chapter 2 answers the question - Do we have a mixed methods research project? Chapters 3 and 4 address the design of the various aspects of such projects - the choice of data types and samples, data collection, the methods of analysis of the various data elements both individually and in combination so as to achieve the full value of the method through effective synthesis. Chapter 5 views the research project as a whole and brings together all the elements discussed in the previous chapters. I highly recommend this book to aspiring mixed methods researchers. 